\title{
Proposed System for Educational Augmented Reality Smart Book
}

\author{
${ }^{1}$ Amira B. Sallow, ${ }^{2}$ Ahmed A. H. Alkurdi, ${ }^{3}$ Zhvan A. Sulaiman \\ 1,2,3Collage of Computer Science and Information Technology, Nawroz University, Duhok, Kurdistan Region - Iraq
}

\begin{abstract}
Smartphones have greatly advanced in the past decade. The first smartphone had very low processing power and memory. Nowadays, smartphones have close to desktop class processors and memory. They are also equipped with high resolution displays. This advancement enables creating and running sophisticated and large graphics applications. Such as Augmented and Virtual reality. Augmented Reality (AR) can be described as a view of the realworld environment extended with computer-generated three-dimensional objects. AR enables placing objects that are designed in a computer to the user environment with fine precision. AR can be largely useful in a variety of fields.

On that note, AR is making great contribution to the field of education in the present time. Studying and learning different subjects can be a dull experience, especially for primary school children. Children nowadays are growing in the internet and technology era. Thus, education must evolve to the technological standard as well. Using AR to teach children can be exciting, motivating and simulating in a way that is much easier and beneficial than reading textbooks.

In this paper, an alphabet book is designed for key stage 1 students. The design is accomplished in Photoshop CC and Maya3D. Afterwards, using Vuforia and Unity3D, the letters are brought to life when a smartphone is pointed at them. A 3D model of the letter is shown along with a 3D example and audio of the example is played.
\end{abstract}

Keywords: Augmented Reality, Vuforia, Maya3D, Unity3D, Education.

\section{Introduction}

Augmented reality (AR) is a technology that enables merging the physical world with objects created by a computer. The advancement of processors, graphics processing units and RAM storage in handheld devices has allowed such technologies to be run seamlessly. AR differs from Virtual Reality (VR) in that AR extends the real-world environment rather than replacing it (Brown, 2014).

AR is being integrated into a variety of fields currently. The technology can enhance and enrich several aspects

Academic Journal of Nawroz University

(AJNU) Volume 8, No 3 (2019).

Regular research paper: Published 31 Aug 2019

Corresponding author's e-mail : ahmad.ala89@gmail.com

Copyright (C2018 ${ }^{1}$ Amira B. Sallow, ${ }^{2}$ Ahmed A. H. Alkurdi,

3Zhvan A. Sulaiman.

This is an open access article distributed under the Creative Commons Attribution License. of life. Especially education, AR can be used in education to ease and simplify the process of teaching, help student grasp complex ideas and theories, excite and simulate student to learn new ideas, and keep student in touch with the technological advancement of the current time. AR enables teachers to show 3D models of an experiment rather than going through the hustle of acquiring special equipment, also it can help primary school pupils in visualizing and comprehension of different subject materials (Mahale \& Yeddu, 2016).

There are several educational AR applications currently, each application is intended for a specific purpose of education. In a research by P. Mahale and S. Yeddu, an application was developed for children such that when pointed at a meaningful word, a 3D object of the example is shown. For example, pointing the phone 
camera at the world Lion, a 3D lion object is shown (Mahale \& Yeddu, 2016). Another example is an application developed in a research by M. Ulas and S. Tasci. The application developed is intended for scientific purposes. It work when pointing it at a $2 \mathrm{D}$ image of the human skeleton. When pointed, the application overlays a 3D skeleton object on the image (Ulas \& Talsci, 2014).

This paper is concerned with developing a model of a smart book. The smart book is intended for primary school students. The book contains all the letters of the Kurdish language. The application works by pointing a smartphone, which has the application installed, at a page of the book. At that point a 3D model of the character is shown with a $3 \mathrm{D}$ example of an object that has the character included. It also plays a sound of the 3D object. This application can help student learn much quicker. It can help simulate students and gain their undivided attention.

This application is implemented in Unity3d, and by utilizing the Vuforia SDK (software development kit). Unity3D is a gaming engine which enables developing desktop, mobile, AR and VR applications (Unity3D, 2018). Vuforia SDK is a plugin that is added to unity to enable the development of marker based AR applications (Vuforia Inc., 2018). The modeling of the different 3D objects and letters had been achieved using Maya3D. Maya3D is a software used for modeling and texturing 3D objects (Autodesk Inc., Maya3D, 2018). Furthermore, Photoshop CC2018 has been used to create texture for the objects designed in Maya.

\section{Organization of the Proposed System}

The proposed system is organized with two Modeling, Creating Vuforia database and scene. Figure 1 show the steps of the proposed system.

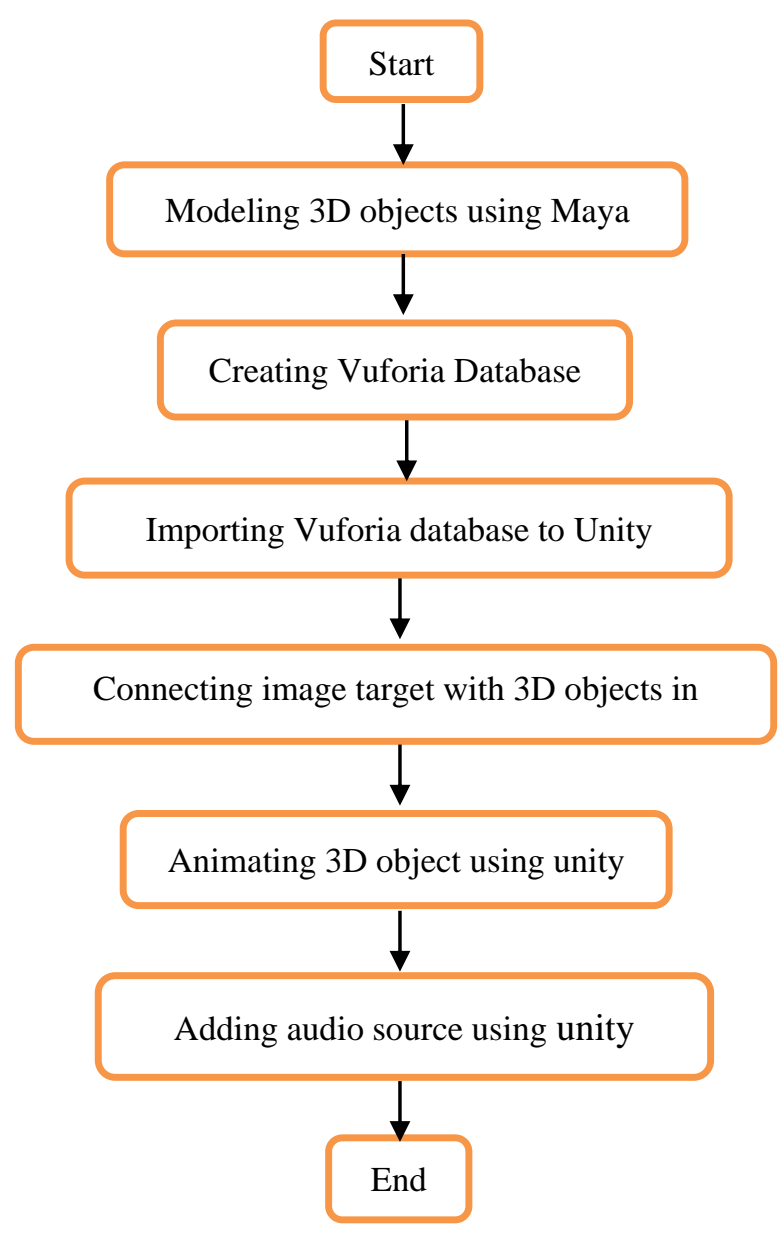

Figure 1: Proposed system flow chart.

\section{Modeling}

Modeling is the process of creating 3D object from primary polygons. In this application, 34 Kurdish language characters were modeled along with 34 objects as examples. See figure 2 for character modeling.

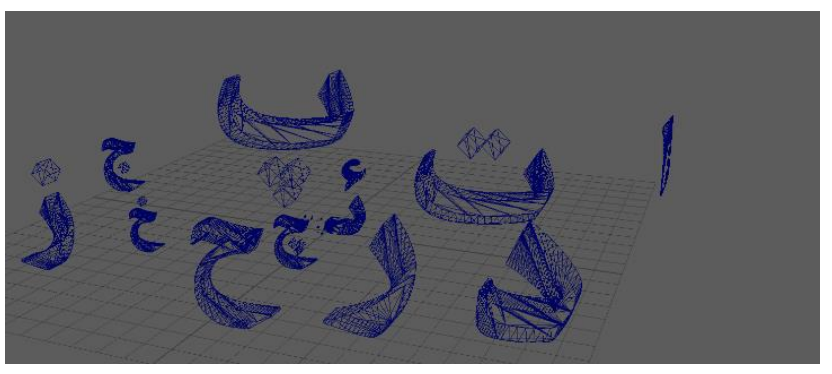

Figure 2: Example modeling of characters. 
Texturing involves adding colors or specific material textures to the different objects. For the textures to be placed on objects, the UV's must be correctly set in the UV editor. UV's are mappings of the texture on the object, i.e. UV's are 2D representations of the surface object mesh (Autodesk Inc., knowledge.autodesk.com, 2018). After setting the UV's, a snapchat of the UV's is taken to Photoshop so that the textures can be correctly mapped to the object. See figure 3 for UV mapping in Photoshop. Also figure 4 for an example of a textured object.

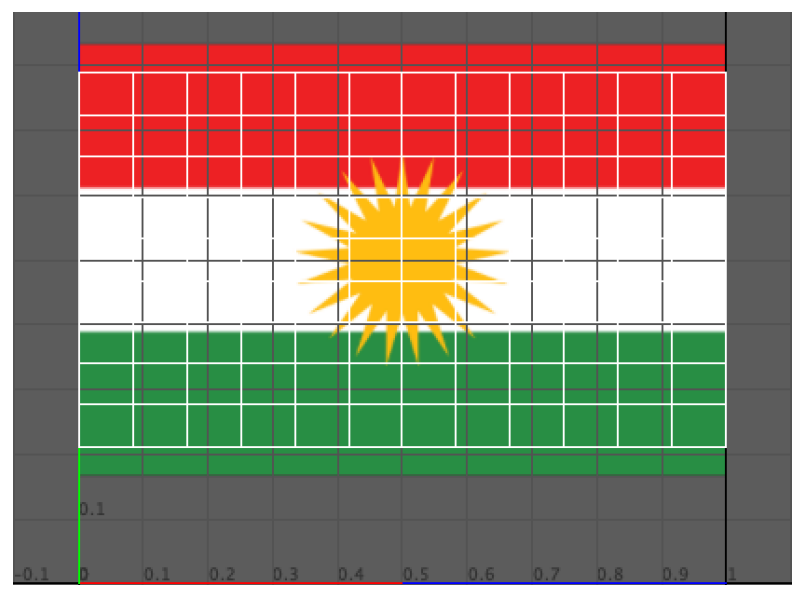

Figure 3: UV Mapping Object.

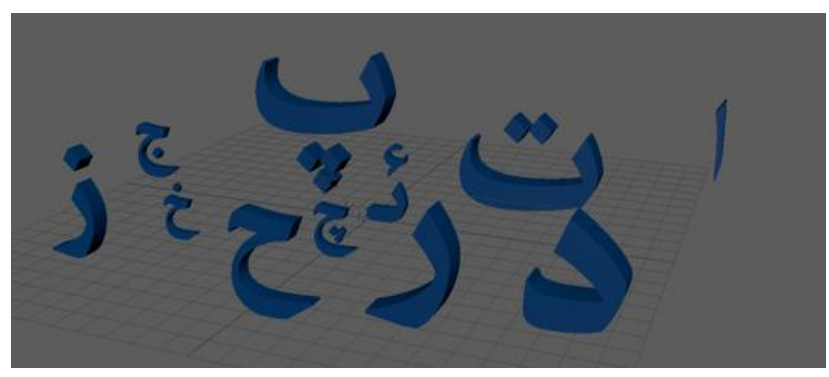

Figure 4: Texturing Characters.

\section{Scene}

The scene of the application has been created in Unity3D. Unity is one of the leading game development IDEs. A range of applications such as, augmented reality, virtual reality, 2 dimensional and 3D dimensional application can be created in unity for a variety of platform (Unity3D, 2018). The application developed relies on Vuforia SDK. Vuforia is a software development kit that enables detecting image targets and overlaying a 3D object over.

\subsection{Vuforia SDK}

Vuforia is an augmented reality development kit which is developed by PTC inc since November 2015. This framework enables tracking image targets and 3D objects. This enables overlaying 3D models over the tracked objects. Vuforia Application Programming Interface (API) in Unity enables creating AR application for iOS and Android devices. Vuforia SDK tracks object and image targets in real time, the orientation and position of the tracked markers are then calculated and 3D models are placed in relation to them in such a manner that the virtual objects are shown as a part of the real world.

Creating a marker in Vuforia involves acquiring images with a reasonable amount of features for detection. Images must be high contrast or medium contrast images, an image of a solid color will not work since it would be hard for the device camera to detect. Afterwards a target database must be created in the Vuforia developers' portal. Then the images must be imported to the portal and the database must be downloaded as a Unity package. By accomplishing that, the images will be set for Unity and the application will be set for detecting the images.

\subsection{Scene Implementation}

After populating the required image targets and importing the database package. All models must also be imported from Maya. Hereafter, animations and sounds are created for each character and example object. The animations and sounds are intended to make the children more excited and enthusiastic with the application. See figure 5 for the scene in Unity Editor. 


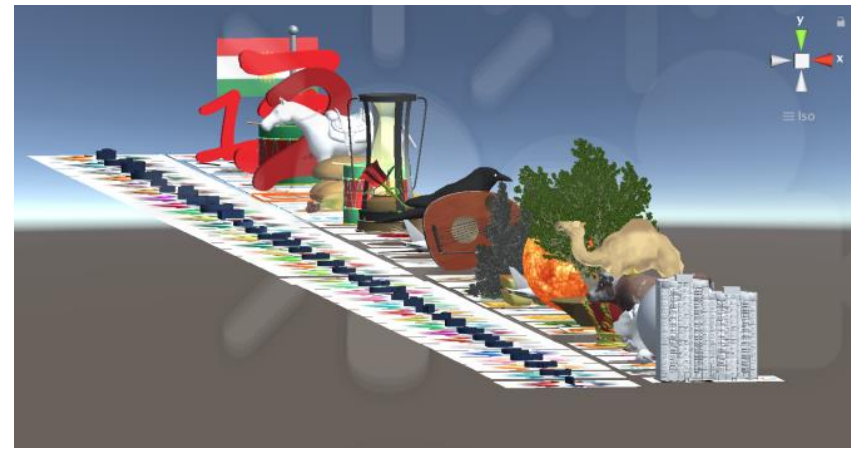

Figure 5: Image Target and Objects in Unity Editor.

\subsection{Animation}

Unity animations can be created to manipulate object position, rotation and scaling at runtime. The proposed application includes several types of animation. Characters are given a rotation animation, while each object is given a different animation, for example, the flag is given a Cloth property and is made to move in an animation simulating a wind movement.

Animations can be created in the animation window. Each animation must be recorded with specific keys at specific time frames. See figure 6 for the animation window.

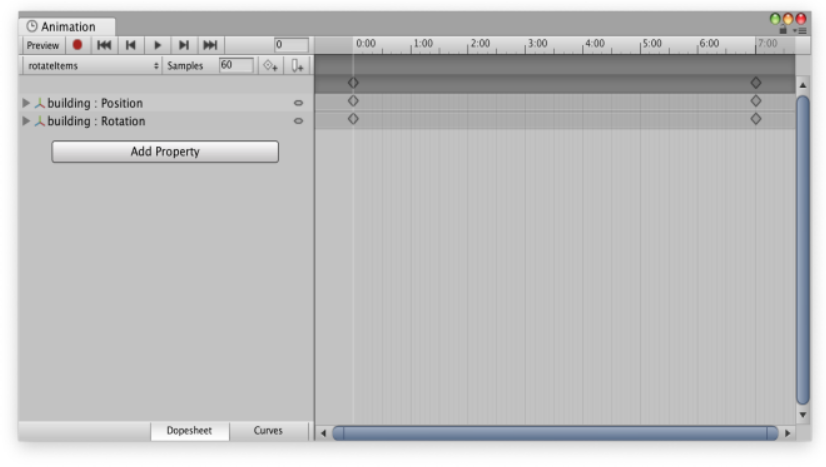

Figure : Recording example object animation.

The animations recorded are saved as a clip. Afterwards, the different clips are attached to the respective objects. The animations start when Vuforia detects the image target and the object is displayed.

\subsection{Audio Source}

An audio source object can be attached to an object to play a sound file. In the proposed application. Each character and each example object has an audio source. Where the characters are pronounces and afterwards the example object sound is played. A specific script has been created for playing the sounds, this is because audio files are played non-stop and all together in the viewed portion of the application. See code below for the audio script.

\section{/ /-----------Begin Sound----------}

public AudioSource soundTarget;

public AudioClip clipTarget;

private AudioSource[] allAudioSources;

/ / function to stop all sounds

void StopAllAudio()

\{

allAudioSources $=$

FindObjectsOfType(typeof(AudioSource))

as

AudioSource[];

foreach (AudioSource audioS in allAudioSources)

\{

audioS.Stop();

\}

\section{/ / function to play sound}

void playSound(string ss)

\{

clipTarget $=($ AudioClip $)$ Resources.Load(ss);

soundTarget.clip = clipTarget;

soundTarget.loop $=$ false;

soundTarget. playOnAwake $=$ false;

soundTarget.Play();

\}

/ /-----------End Sound-------------

\section{Results}

Children nowadays live in a technological age. There 
are different tablet devices for children, almost all children have their personal tablet. Thus studying with boards and chalk must be really tedious to them. They almost feel like living in two different ages, one where there is technology and internet, and the other where it's black and white. That being said, it is of high importance that children should be taught in the same way they live. Bringing technologies like AR to their school can help keep them keen and interested in learning and focusing on the matters at hand. Naturally children concentrate at colorful objects. Thus, teaching them in such a manner can be easier for the teacher, and also easier for the children to learn and memorize the different character and their shape.

The proposed application helps first year students learn the Kurdish language characters in a way that has never been used before. Showing them live animations of the examples provided and the characters moving. This is an essential step towards the future of education. See figure $7 \mathrm{a}$ and $7 \mathrm{~b}$ for the application at run time.

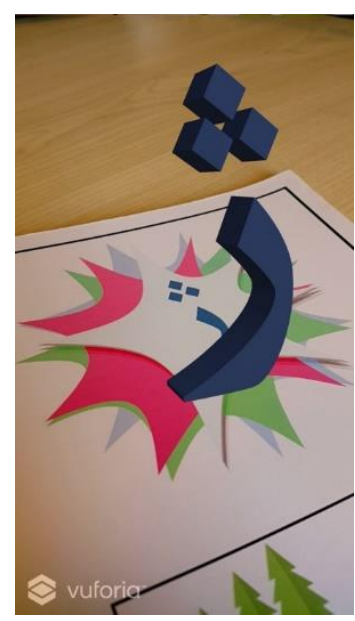

Figure 7a: Character Model at Run Time.

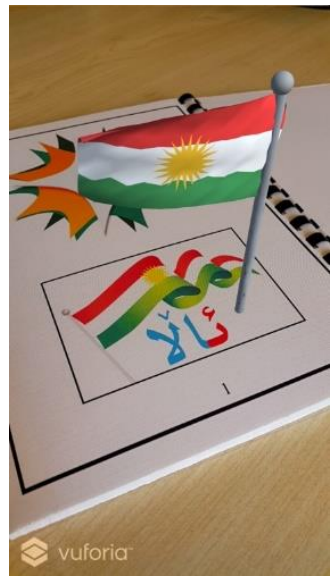

Figure 7b: Object Model at Run Time.

\section{Conclusion}

To conclude, the availability of high-end tablets and devices has enabled creating such application for the public. Technologies like AR and VR can change the future in ways that are beyond imagination. Specially in education, with the advancement of technology and it's integration into people's lives. It must also be integrated into education to help children prepare for the real world after.

In this paper, a model of a smart book has been created to help primary school student learn in a more advanced and enthusiastic method. This can ease the work teachers have to put through to educate the future generation.

\section{Bibliography}

1. Autodesk Inc. (2018). knowledge.autodesk.com. Retrieved from autodesk.com: https://knowledge.autodesk.com/support/maya/ learn-

explore/caas/CloudHelp/cloudhelp/2018/ENU/ Maya-Modeling/files/GUID-FDCD0C68-24964405-A785-3AA93E9A3B25-htm.html

2. Autodesk Inc. (2018). Maya3D. Retrieved from Autodesk:

https://www.autodesk.com/products/maya/over view 
3. Brown, K. (2014). Prelude - An Augmented Reality iOS Application for Music Education. Bowling Green State University.

4. Mahale, P., \& Yeddu, S. (2016). Android-based Augmented Reality to Enhance Education System. International Journal of Computer Applications, 18 - 21.

5. Ulas, M., \& Talsci, S. M. (2014). A Review About Augmented Reality Tools and Developing A Virtual Reality Application Based On Education. Academic Journal Of Science, 139 - 146.

6. Unity3D. (2018). Unity3d. Retrieved from Unity3D: https://unity3d.com/unity

7. Vuforia Inc. (2018). Vuforia. Retrieved from Vuforia: https://www.vuforia.com 